\title{
Source Reconstruction with the Electromagnetic Space Kurtosis and Time Kurtosis in the Time-Reversal Technique
}

\author{
Xiao-Yao Feng, Graduate Student Member, IEEE, Zhizhang (David) Chen, Fellow, IEEE
}

\begin{abstract}
In our previous study, a concept of electromagnetic kurtosis for time-reversal source reconstruction was presented. From a two-step process, the electromagnetic kurtosis can first identify the excitation time instants then localize the sources locations with time-reversal technique. In this paper, we extend the concept of the electromagnetic kurtosis from two different perspectives. The proposed space kurtosis and time kurtosis in the electromagnetic time-reversal process can directly reconstruct excitation time instants and spatial locations of sources respectively. The newly proposed time kurtosis takes the advantages of time-domain computational electromagnetics methods. Its algorithm with the threshold technique simplifies the procedures of multiple sources localization. Numerical experiments are conducted to verify the effectiveness of the presented algorithm.
\end{abstract}

Index Terms - computational electromagnetics (CEM), finitedifference time-domain (FDTD) method, kurtosis, source reconstruction, time-reversal (TR).

\section{INTRODUCTION}

$\mathrm{T}$ he time-reversal (TR) technique has been used to solve inverse problems in various fields, such as acoustics [1]-[3] and communication systems [4]. In electromagnetics and microwave engineering, TR is applied to geolocation [5], radar imaging [6], clinical use with microwave imaging [7], [8], and other problems. One possible important TR application is source reconstruction/localization [9]-[16], which is critical in electronic system designs: locating the transient interference sources for electromagnetic compatibility (EMC); such a source localization is generally hard to be solved by direct measurements and testing.

However, much work on the electromagnetic TR source reconstruction so far has only considered the identification of sources excited at the same initial time or simple experiment conditions without noises. These limitations impede the TR technique from being applied to complicated realistic scenarios.

On the other hand, kurtosis is a concept often used in statistical data analysis which quantifies the proportion and "sharpness" of the outliers in a random variable distribution [17], [18]. The larger the kurtosis value, the larger proportion and values of the outliers in a data set. Recent researches have shown that the use of kurtosis is not only restricted in statistics, but applied to other fields like communication systems [19], [20] (and references therein). There are different ways of estimating the kurtosis from a finite sampled data set with varying terminologies, one is called the sample kurtosis. It is a scaled version of the ratio between the fourth central moment and the square of the second central moment. Inspired by these ideas, we introduced the electromagnetic kurtosis and incorporated it into the electromagnetic TR technique in our previous work [21].

As our previous work only laid a groundwork of kurtosis use in TR source reconstruction, this paper try to develop it. Inspired by the relativity between space and time in mathematical physics, the electromagnetic time kurtosis is proposed. Together with the kurtosis, which is now re-defined as the electromagnetic space kurtosis, presented in our previous work, the concept of the electromagnetic kurtosis is developed. The electromagnetic time kurtosis utilizes the advantages of the time-domain computational electromagnetics method in solving transient electromagnetic problems, thus leading to a simpler process when conducting source reconstruction with the TR technique.

We organize the paper as follows. In section II, we introduce two kinds of the electromagnetic kurtosis, and use examples to demonstrate their advantages in characterizing electromagnetic field distribution respectively. In section III, we briefly present the mathematical analysis of electromagnetic TR technique. We then incorporate the two kinds of electromagnetic kurtosis into the process of TR source reconstruction and compare them. In section IV, we develop the threshold technique of the electromagnetic time kurtosis and conduct numerical experiments of multiple sources reconstruction. The results demonstrate the effectiveness and efficiency of the proposed algorithm. Finally, in section V, we make conclusions.

\section{Electromagnetic Space Kurtosis AND Time KuRtosis}

In this part, we will first introduce the concepts of two kinds of electromagnetic kurtosis for characterizing electromagnetic field distribution, then run numerical experiments to verify their feasibility and capability.

Consider a time-varying electric (or magnetic) field $E$ that is sampled at a spatial position $(i \delta, j \delta, k \delta)$ and at a time instant $t=$ $n \Delta t$ with the space increment being $\delta$ and time increment being 
$\Delta t$. Denote this sampled electric (or magnetic) field at the position $(i \delta, j \delta, k \delta)$ as $E(i, j, k, n)$ with $n$ referred to time $t=n \Delta t$. Assume that the total number of the samples in space is $L$ and in time is $N$. When the finite-difference time-domain (FDTD) method is applied, the node $(i, j, k)$ is the FDTD grid position. With these notations, we introduce two kinds of the electromagnetic kurtosis as described below.

\section{A. The Electromagnetic Space Kurtosis}

In our previous work, the electromagnetic kurtosis was proposed to identify source excitation time intants [21]. As it is a measure of the space distribution of an electric (or magnetic) field at a specific time step $n$, we re-write it here and define the electromagnetic space kurtosis (hereinafter referred to as "space kurtosis") as follows:

$$
\beta_{s}(n)=\frac{m_{4}}{m_{2}^{2}}=\frac{\frac{1}{L} \sum_{i} \sum_{j} \sum_{k}\{E(i, j, k, n)-\bar{E}(n)\}^{4}}{\left[\frac{1}{L} \sum_{i} \sum_{j} \sum_{k}\{E(i, j, k, n)-\bar{E}(n)\}^{2}\right]^{2}} .
$$

$m_{4}=\frac{1}{L} \sum_{i} \sum_{j} \sum_{k}\{E(i, j, k, n)-\bar{E}(n)\}^{4}$ is the fourth central moment of the field sample set in space, and $m_{2}=\frac{1}{L} \sum_{i} \sum_{j} \sum_{k}\{E(i, j, k, n)-\bar{E}(n)\}^{2}$ is the second central moment of the sample set. $\bar{E}(n)$ is the field value averaged over all the FDTD grids at the time step $n$. Note that in (1), the triple summation $\sum_{i} \sum_{j} \sum_{k}(\ldots)$ is taken with respect to all the FDTD grid points in space. Thus the space kurtosis $\beta_{s}$, which characterizes the field distribution in space as its name suggests, is then a function of the time step (or instant) $n$.

In statistics and probability theory, the sample kurtosis is used as a measure of the "sharpness" of outliers in a random variable distribution [18]. With the space kurtosis of (1), the shape of a electromagnetic field distribution in space can be quantified. The larger the space kurtosis values, the larger field concentrations of a distribution in space; such large field values correspond to potential transient sources. In other words, by checking the space kurtosis values of a field distribution at each time step (or instant), we can identify the time instants of potential sources excitation (i.e., when large space kurtosis values appear).

\section{B. The Electromagnetic Time Kurtosis}

Comparing to the concept of kurtosis in statistics, it is not hard to understand how the proposed space kurtosis characterizes a field distribution in space by analogy. Furthermore, a successful transient source reconstruction should obtain field focus not only in space but also in timedomain because of its uniqueness. Thus we change the perspective from space to time; for an electric (or magnetic) field distribution sampled at a specific spatial node $(i, j, k)$, we introduce the electromagnetic time kurtosis (hereinafter referred to as "time kurtosis") as follows:

$$
\beta_{t}(i, j, k)=\frac{\frac{1}{N} \sum_{n}\{E(i, j, k, n)-\bar{E}(i, j, k)\}^{4}}{\left[\frac{1}{N} \sum_{n}\{E(i, j, k, n)-\bar{E}(i, j, k)\}^{2}\right]^{2}} .
$$

Here $\bar{E}(i, j, k)$ is the field value averaged over all the FDTD time steps at the spatial node $(i, j, k)$ and the summation $\sum_{n}(\ldots)$ is taken with respect to all the time steps. Therefore, the time kurtosis $\beta_{t}$ can characterize the field distribution in time-domain, especially when large kurtosis values occur; that is, by checking the large values of time kurtosis in the solution domain (in space), we can localize transient sources.

In the following subsection, we conduct a numerical experiment to verify the capability of the space and time kurtosis to capture the source excitation time instant and location respectively.

\section{Numerical Verification of the Space Kurtosis and the Time Kurtosis to Identify the Source Excitation Time and Location}

With the FDTD method, we conduct a numerical experiment to verify the capability of the space kurtosis and the time kurtosis to identify transient source excitation time and location respectively. For simplicity, we consider a TM-to-z wave twodimensional cavity with $30 \times 30$ cells size. In our numerical experiment, we place a single impulse source at a random selected node $(14,17)$ and excite it at a random selected time step of $n=2000$. The space kurtosis and the time kurtosis are computed with respect to (1) and (2), and the results are shown in Fig. 1.

Fig. 1(a) shows the electric field signal at the node $(14,17)$. We can find that the source is excited at the $2000^{\text {th }}$ time step. From Fig. 1(b) we can find that a maximum does occur at the $2000^{\text {th }}$ time step; this time instant corresponds to the source excitation time in Fig. 1(a). Before the $2000^{\text {th }}$ time step, the electric field is not excited, and the space kurtosis does not exist. After the $2000^{\text {th }}$ time step, the electromagnetic field propagates inside the cavity and its energy spread to every nodes; thus the space kurtosis varies with time in relatively small values.

Fig. 1(c) shows the field distribution at the $2000^{\text {th }}$ time step. The E-bar on the right denotes the field intensity. The bright yellow dot located at the node $(14,17)$ denotes the source location. Fig. 1(d) shows the results of the computed time kurtosis of (2). The location of the bright yellow dot is where the maximum value of the time kurtosis occurs. This yellow dot corresponds to the location of the source in Fig. 1(c). 


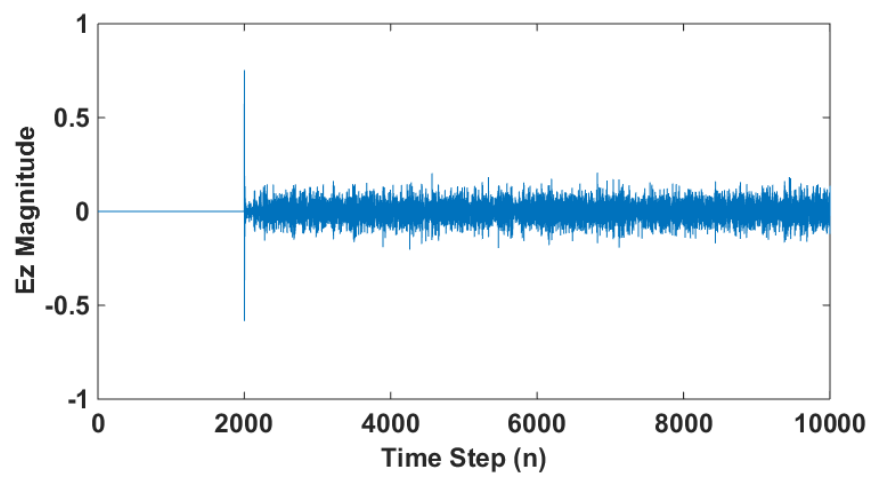

(a)

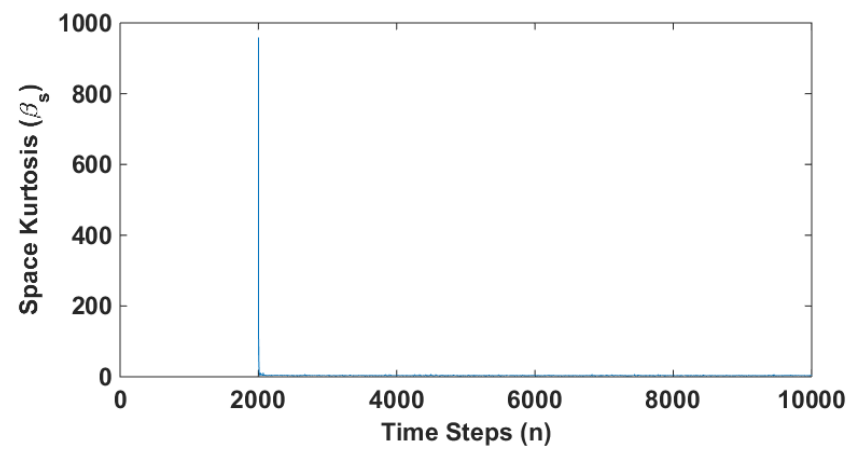

(b)

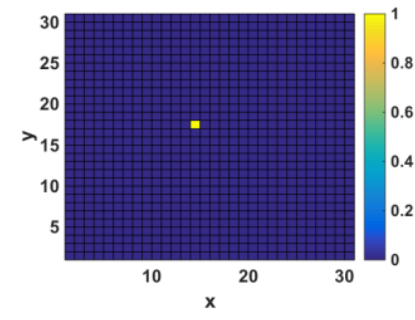

(c)

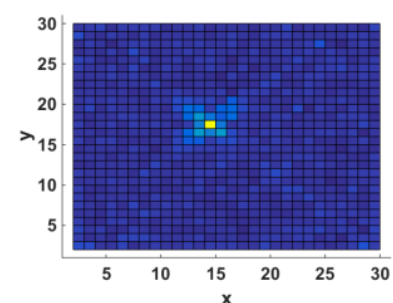

(d)
Fig. 1. FDTD simulation of a 2D cavity with an impulse source excitation. (a) The electromagnetic signal recorded at the node $(14,17)$. (b) The computed space kurtosis value versus time. (c) The space distribution of electromagnetic field at the $2000^{\text {th }}$ time step. (d) The distribution of the time kurtosis.

In summary, the numerical experiment demonstrates the functions of the two kinds of electromagnetic kurtosis. On the one hand, we can use the space kurtosis of (1) to identify the source excitation time instant by finding the time step when the space kurtosis value reaches the maximum. On the other hand, we can apply the time kurtosis to locating transient source directly from the time kurtosis distribution in space. In the next part, we will incorporate these two kinds of electromagnetic kurtosis into the TR technique to reconstruct sources more efficiently.

\section{TIME-REVERSAL SOURCE RECONSTRUCTION WITH THE SPACE KURTOSIS AND THE TIME KURTOSIS}

In this section, we will first present the mathematical analysis of the electromagnetic TR technique from the view of discretetime signal processing. Then we will introduce the TR process with the space kurtosis, which was presented in our previous study. Finally, we will incorporate the time kurtosis into the TR process and compare it with that of the space kurtosis.

\section{A. The Electromagnetic Time-Reversal Technique}

A conventional electromagnetic TR process or algorithm includes two phases [12]:

Phase \#1: The first is the forward simulation or measurement that records the field responses generated by unknown sources.

Phase \#2: The second is the backward simulation or TR computation that reconstructs those sources.

In the phase $\# 1$, we record the field responses in a certain period at preselected spatial positions with proper sensors (time-reversal mirrors (TRM) [22]). In a real-world scenario, an actual hardware experiment is conducted, and field sensors are placed inside a cavity. In our case, we do not carry out the measurements or testing but use the FDTD simulations instead; we use the FDTD method to emulate the cavity and record the signals at these TRM locations during the (FDTD) forward simulations. Since the cavity is ergodic, electromagnetic wave will pass through every location inside it equally in a statistically averaged sense; thus the TRM locations will not affect the TR results much.

Suppose we have $Q$ unknown transient (impulse) sources $x_{q}[n]$ at $Q$ locations; they may be excited at different unknown time. We can express them in the form of:

$$
x_{q}[n]=a_{q} \delta\left[n-n_{q}\right], q=1,2, \ldots, Q,
$$

where $a_{q}$ and $n_{q}$ are the amplitude and the excitation time step of the $q$ th source.

The fields generated by the $Q$ sources of (3) will reflect and propagate to the preselected TRM locations and are recorded there. Assume that we have $P$ TRM (sensors), the signal recorded at the $p$ th sensor can then be expressed as:

$$
y_{p}[n]=\sum_{q=1}^{Q} a_{q} h_{p q}\left[n-n_{q}\right], p=1,2, \ldots, P ; n=0,1, \ldots, N,
$$

where $h_{p q}[n]$ is the impulse response at the $p$ th sensor due to the impulse excitation at the unknown $q$ th source location. Here $N$ is the total number of the time steps of the forward simulation (measurement).

Once the signals are recorded at $P$ sensors, we complete the phase \#1 and move on to the phase \#2.

In the phase \#2, we first time-reverse the received signal at the $p$ th sensor (hence we call the technique the TR technique), and it become:

$$
y_{p}^{r}[n]=y_{p}[N-n]=\sum_{q=1}^{Q} a_{q} h_{p q}\left[N-n+n_{q}\right] .
$$

Then we re-inject the time-reversed signals into the solution domain at all sensors and run the backward simulation. The output signal at the original $q$ th source node can be computed as:

$$
s_{q}[n]=\sum_{p=1}^{P} h_{q p}[n] \otimes y_{p}^{r}[n]=\sum_{p=1}^{P} \sum_{m=0}^{n} h_{q p}[m] y_{p}^{r}[n-m] .
$$

Theoretically, at the time steps $n=N-n_{q}$ in the phase \#2, the output signal of (6) $s_{q}\left[N-n_{q}\right]$ is larger than the fields at any other non-source locations, contributing to the field concentrations at the original $Q$ source locations [21]. 


\section{B. Typical Process of Time-Reversal Source Reconstruction with the Space Kurtosis}

In our previous work, the space kurtosis of (1) was incorporated into the phase \#2 of the TR process [21]. As described in Section II, the space kurtosis can determine the source(s) excitation time instant(s) by identifying the maximum (local maxima) value(s) of itself. Therefore, we can then plot the space distributions of electromagnetic field at these time steps (or instants) and localize and reconstruct the sources spatially. A numerical example is shown below.

We use the cavity of $30 \times 30$ FDTD cells again for verification. We place a single impulse source at the node (23, $19)$ and three TRM at $(13,5),(3,19)$, and $(11,7)$ nodes, respectively. The single impulse is excited at the $1000^{\text {th }}$ time step (i.e., $Q=1, n_{1}=1000$ ) and we run the forward simulation of the phase $\# 1$ up to the $10000^{\text {th }}$ time step (i.e., $N=10000$ ). After the phase \#1, we carry out TR simulation of the phase \#2 and compute and record the space kurtosis at every time step. The results are shown in Fig. 2.

Fig. 2(a) is the computed space kurtosis over the whole backward simulation (i.e., the phase \#2). Comparing to the Fig. 1(b), the space kurtosis results in backward simulation act a bit different. The space kurtosis in this phase \#2 has certain values all over the whole period and presents two local maxima: one is at the beginning of the backward simulation, and the other is at the time step corresponding to the original source in the phase \#1. The first maximum does not correspond to the original source; it occurs at the beginning of the backward simulation and corresponds to the time-reversed signals of (5) that are reinjected into the cavity (as the sources of the phase \#2). The exact time instant of the second local maximum is the $9000^{\text {th }}$ time step, which matches the predicted one $\left(N-n_{1}=\right.$ $10000-1000=9000)$. Once we identify the $9000^{\text {th }}$ time step, we plot the space distribution of the field at the $9000^{\text {th }}$ time step; Fig. 2(b) shows this space distribution at the $9000^{\text {th }}$ time step obtained with the backward simulation of the phase \#2. A bright yellow dot locates at the node $(23,19)$, which is exactly the same location of the original source; this means that we reconstruct the source with the space kurtosis successfully.

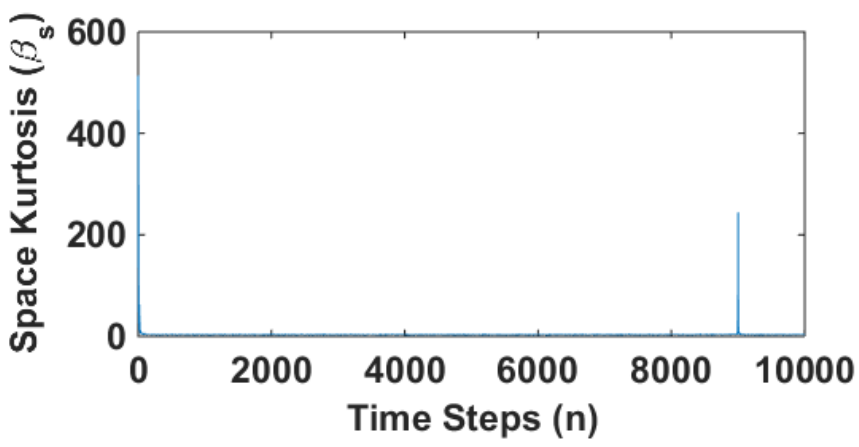

(a)

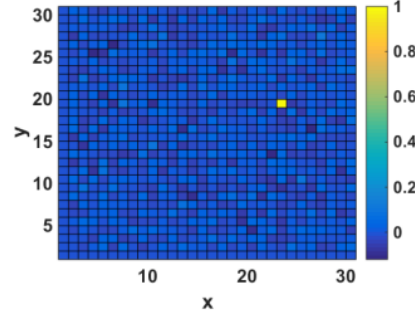

(b)

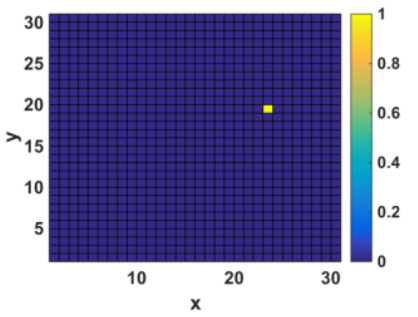

(c)
Fig. 2. The TR reconstruction of a single impulse source. (a) The computed space kurtosis in the phase \#2. (b) The field distribution at the time the space kurtosis reaches the second local maximum and the reconstructed source. (c) The original source and related field distribution.

Now we can conclude that the process of TR source reconstruction with the space kurtosis contains two steps:

i) Indentify the time instant(s) when the space kurtosis reaches the valid local maxima in the TR simulation of the phase \#2;

ii) Plot the space distribution of the field at those indentified time instant(s) in the phase \#2 and localize the source(s).

\section{Incorporation of the Time Kurtosis in the Time-Reversal Process}

In EMI/EMC applications, we tend to care more about the locations of transient interference sources than their excitation time. Although the proposed space kurtosis is capable of reconstructing source locations, the summarized process above shows that the indentification of excitation time instants in the phase \#2 is prerequisite. However, by introducing the time kurtosis of (2) in the TR process, the spatial location of the transient source can be directly reconstructed.

Fig. 3 shows an example of the TR source reconstruction with the time kurtosis. We place a single impulse source at the node $(6,5)$ in the same $30 \times 30$ FDTD cavity. We do not need to know when the source is excited. The calculated time kurtosis results are shown in Fig. 3(a). As the time kurtosis values of (2) vary in the space, the distribution of the computed time kurtosis seems similar to the space distribution of electromagnetic field. The location of the bright yellow dot is the node $(6,5)$, which matches that of the original source.

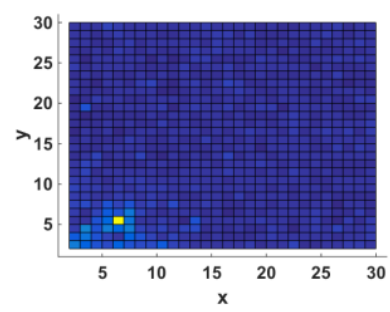

(a)

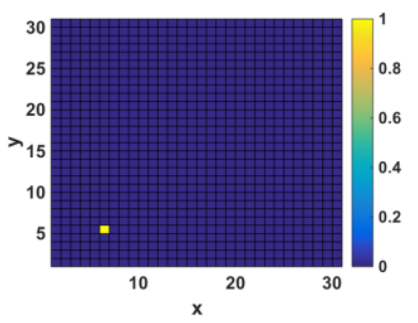

(b)
Fig. 3. The TR reconstruction of a single impulse source with the time kurtosis. (a) The computed time kurtosis in the phase \#2. (b) The original source and related field distribution.

From the example of Fig. 3, TR source reconstruction only need one step: plot the space distribution of the time kurtosis of (2) computed in the phase \#2. It simplifies the process of source reconstruction comparing with that of the space kurtosis. Furthermore, after indentifying the maximum value, the time 
kurtosis directly demonstrates the results while the method with the space kurtosis has to discard invalid local maxima at first. In other words, the reconstructed source location $\left(i_{0}, j_{0}\right)$ can be determined directly by:

$$
\left(i_{0}, j_{0}\right)=\arg \max _{(i, j)} \beta_{t}(i, j) .
$$

In three-dimensional cases, this direct algorithm can be written as:

$$
\left(i_{0}, j_{0}, k_{0}\right)=\arg \max _{(i, j, k)} \beta_{t}(i, j, k) .
$$

\section{Time-ReVersal Localization of MultiPle Sources WITH THE TIME KURTOSIS}

With the proposed time kurtosis of (2), we can simplify the process of source reconstruction with TR technique. However, the experiment above works with a simple case where only one single impulse source exists in the solution domain. In complex scenarios, multiple sources may exist at different locations and unknown time instants. In this section, we will extend the feasibility of the time kurtosis method and carry out experiments with the threshold technique to solve this problem.

\section{A. The Threshold Technique of the Time Kurtosis}

In the example of Fig. 3, we assume that only one source exists. However, in real applications, we do not have much priori knowledge of the sources, including excitation time instants, sources locations and number of sources. When multiple sources are excited at different locations, the algorithm of (7) and (8) may not work well. Therefore, by referring to our previous study, the threshold technique is developed.

With the components of our numerical experiments above, we carry out the following computation to determine the threshold value of the time kurtosis. The time kurtosis is computed on a time-domain signal with one single unit impulse excited at one time step and random values at the rest of time. The random signal values are used to emulate noises at other non-source time instants; they are of normally distributed random values with a magnitude between 0 and $E_{n}$. We repeat the computation with different strengths of $E_{n}$, and the results are shown in Fig. 4.

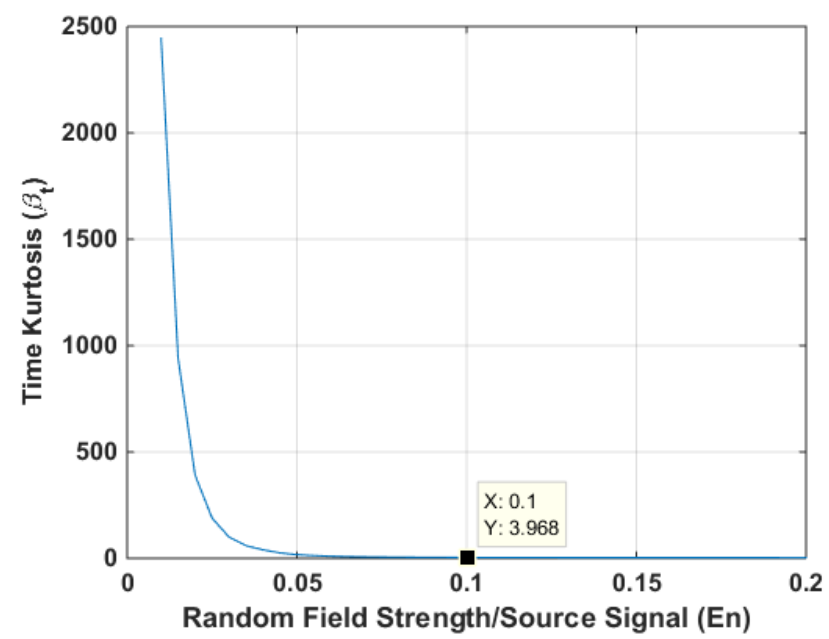

Fig. 4. The relationship between the time kurtosis and the ratio of random fields' strength to the impulse source strength.

Fig. 4 shows the computed time kurtosis versus the ratio of the random fields' strength to the impulse source strength; the ratio is equal to $E_{n}$. As seen, when the ratio increases (i.e., stronger noise), the time kurtosis decreases.

We take $E_{n}=0.1$ as the threshold point as it amounts to that the maximum strength of the random noises is $10 \%$ (or $-20 \mathrm{~dB}$ ) of the source. We consider it as the breaking point to distinguish the valid kurtosis maxima from others. At $E_{n}=0.1, \beta_{t}=$ 3.968; thus $B=\beta_{t}=3.968$ is used as the threshold value of the time kurtosis: any kurtosis value above it will be considered as the valid local kurtosis maxima for sources locations identification. In other words, all the reconstructed sources locations $(i, j)$ in the TR backward simulation (the phase \#2) can be computed by the algorithm below:

$$
(i, j)=\left\{\left(i_{q}, j_{q}\right) \mid \forall \beta_{t}\left(i_{q}, j_{q}\right)>B\right\}, q=1,2, \ldots
$$

Again, in three-dimensional cases, the set of reconstructed sources locations $(i, j, k)$ can be calculated by:

$$
(i, j, k)=\left\{\left(i_{q}, j_{q}, k_{q}\right) \mid \forall \beta_{t}\left(i_{q}, j_{q}, k_{q}\right)>B\right\}, q=1,2, \ldots
$$

\section{B. Time-Reversal Localization of Multiple Sources}

We now apply the proposed threshold technique to verify the effectiveness of (9) and (10) in reconstructing multiple sources with the time kurtosis. Again, we consider the $30 \times 30$ FDTD cavity with three TRM at $(13,5),(3,19)$, and $(11,7)$ nodes. Two impulse sources are $x_{1}[n]=\delta[n-100]$ located at the center node $(15,15)$ and $x_{2}[n]=\delta[n-300]$ located at a random selected node $(19,6)$. The computation results are discussed below with simulation results shown in Fig. 5

By conducting the TR method, received signals at three TRM are re-injected into the cavity in the phase \#2. After computing the time kurtosis of (2) and using the algorithm of (9), the time kurtosis of two nodes are found: $\beta_{t}(15,15)=5.98$ and $\beta_{t}(19,6)=5.78$. These two nodes correspond exactly to the two original sources locations, which means that those multiple sources are reconstructed successfully. On the other hand, we can find from the Fig. 5(a) that the two bright yellow dots match with those in Fig. 5(b) and (c) respectively; this also verifies our computation.

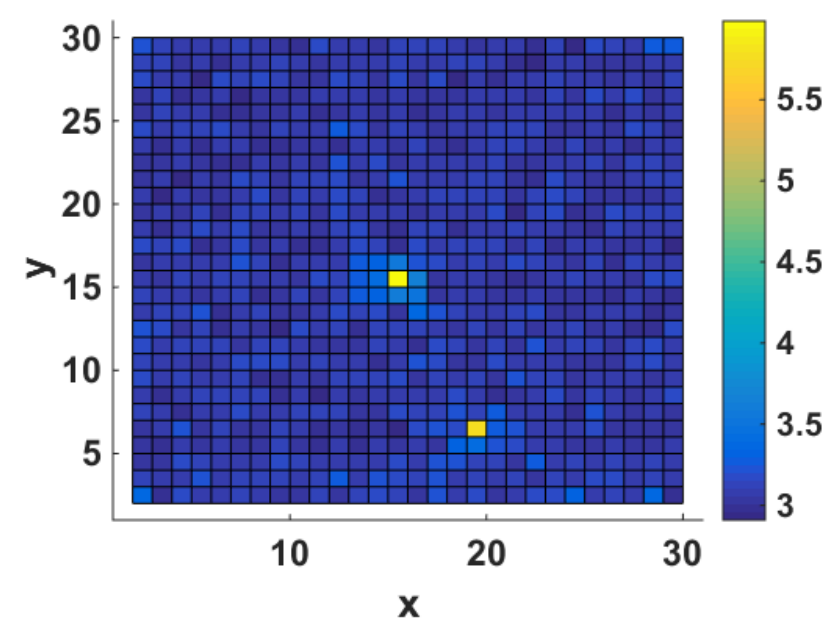

(a) 


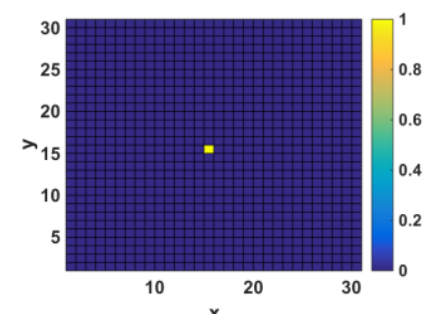

(b)

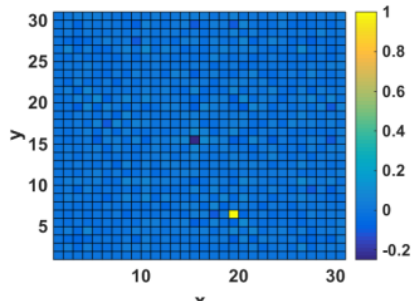

(c)
Fig. 5. The TR reconstruction of multiple sources with the time kurtosis. (a) The space distribution of the computed time kurtosis in the TR backward simulation (the color-bar on the right denotes the value of the time kurtosis). (b) The first source excited at the $100^{\text {th }}$ time step and related field distribution (the E-bar on the right denotes the magnitude of the electric field). (c) The second source excited at the $300^{\text {th }}$ time step and related field distribution.

\section{CONCLUSIONS}

Our previous study provided a groundwork of introducing the kurtosis into the electromagnetic TR method. Here we extend the concept of the electromagnetic kurtosis and summarize them in this paper:

1) The previously introduced electromagnetic kurtosis is re-defined as the space kurtosis for electromagnetic TR source reconstruction. As its name suggests, the space kurtosis is used to quantitively describe the space distribution of electromagnetic field. If a large space kurtosis value occurs at a specific time instant, it means that there exists a field concentration in the space. Therefore, the space kurtosis is a useful tool in determining the source excitation time instants with the TR technique.

2) Different from the view of space, we introduce another kind of electromagnetic kurtosis called the time kurtosis in this paper. It numerically characterizes the signal in time-domain at a certain location. This means that when we gather the values of the time kurtosis in the whole solution domain in TR process, those positions with large time kurtosis values are possible sources locations.

In order to verify the feasibility and capability of the two kinds of electromagnetic kurtosis, we then carry out numerical experiments with FDTD TR. When comparing with the TR source reconstruction process of the space kurtosis, the results show that the proposed time kurtosis works more efficiently on determining the source location. By developing the threshold technique of the time kurtosis, we can also deal with multiple sources reconstruction problems easily; numerical results verify the algorithm of (9) and (10).

In summary, this paper is a substantial extension of our previous work. We summarize the electromagnetic kurtosis in FDTD TR technique and enrich its theoretical basis. This paper only presents our initial work towards practical applications of the TR technique with the electromagnetic kurtosis; further developments will be reported in the near future.

\section{REFERENCES}

[1] M. Fink, "Time reversal of ultrasonic fields. I. Basic principles," IEEE Trans. Ultrason., Ferroelectr., Freq. Control, vol. 39, no. 5, pp. 555-566, Sept. 1992.
[2] F. Wu, J. Thomas and M. Fink, "Time reversal of ultrasonic fields. II. Experimental results," IEEE Trans. Ultrason., Ferroelectr., Freq. Control, vol. 39, no. 5, pp. 567-578, Sept. 1992.

[3] D. Cassereau and M. Fink, "Time-reversal of ultrasonic fields. III. Theory of the closed time-reversal cavity," IEEE Trans. Ultrason., Ferroelectr., Freq. Control, vol. 39, no. 5, pp. 579-592, Sept. 1992.

[4] C. Oestges, A. Kim, G. Papanicolaou and A. Paulraj, "Characterization of space-time focusing in time-reversed random fields," IEEE Trans. Antennas Propag., vol. 53, no. 1, pp. 283-293, Jan. 2005.

[5] H. Karami, M. Azadifar, A. Mostajabi, M. Rubinstein and F. Rachidi, "Numerical and experimental validation of electromagnetic time reversal for geolocation of lightning strikes," IEEE Trans. Electromagn. Compat., vol. 62 , no. 5 pp. $2156-2163$, Oct. 2020.

[6] D. Liu, G. Kang, L. Li, Y. Chen, S. Vasudevan, W. Joines, Q. Liu, J. Krolik and L. Carin, "Electromagnetic time-reversal imaging of a target in a cluttered environment," IEEE Trans. Antennas Propag., vol. 53, no. 9, pp. 3058-3066, Sept. 2005.

[7] P. Kosmas and C. Rappaport, "Time reversal with the FDTD method for microwave breast cancer detection," IEEE Trans. Microw. Theory Techn., vol. 53, no. 7, pp. 2317-2323, Jul. 2005.

[8] P. Kosmas and C. Rappaport, "FDTD-based time reversal for microwave breast cancer detection-localization in three dimensions," IEEE Trans. Microw. Theory Techn., vol. 54, no. 4, pp. 1921-1927, Jun. 2006.

[9] W. Fan, Z. Chen and W. Hoefer, "Source reconstruction from wideband and band-limited responses by FDTD time reversal and regularized least squares," IEEE Trans. Microw. Theory Techn., vol. 65, no. 12, pp. 47854793, Dec. 2017.

[10] W. Fan and Z. Chen, "A condition for multiple source reconstructions with the time-reversal methods," in IEEE MTT-S Int. Microw. Symp. Dig., San Francisco, CA, USA, May 2016, pp. 1-4.

[11] W. Fan and Z. Chen, "A new time reversal method with extended source locating capability," in IEEE MTT-S Int. Microw. Symp. Dig., Honolulu, HI, USA, June 2017, pp. 704-706.

[12] J. Liang, Z. Chen, J. Wang, H. Zhao, C. Peng and Z. Liu, "Time-reversal reconstructions of clustered sources and diagnosis of faulty antenna elements in three dimensions," in IEEE MTT-S Int. Microw. Symp. Dig., Boston, MA, USA, June 2019, pp. 114-116.

[13] X. Feng, Z. Chen and J. Liang, "The entropy technique for the time-reversal source reconstruction," in IEEE MTT-S Int. Microw. Symp. Dig., Los Angeles, CA, USA, Aug. 2020, pp. 158-160.

[14] H. Karami, M. Azadifar, A. Mostajabi, M. Rubinstein, H. Karami, G. Gharehpetian and F. Rachidi, "Partial discharge localization using time reversal: application to power transformers," Sensors, vol. 20, no. 5, pp. $1419,2020$.

[15] A. Mostajabi, H. Karami, M. Azadifar, A. Ghasemi, M. Rubinstein and F. Rachidi, "Single-sensor source localization using electromagnetic time reversal and deep transfer learning: application to lightning," Nature Scientific Reports, vol. 9, pp. 17372, 2019.

[16] H. Karami, M. Azadifar, A. Mostajabi, P. Favrat, M. Rubinstein and F. Rachidi, "Localization of electromagnetic interference sources using a time reversal cavity, "IEEE Trans. Ind. Electron., vol. 68, no. 1, pp. 654662, Jan. 2021.

[17] K. Balanda, and H. MacGillivray, "Kurtosis: a critical review," The American Statistician, vol. 42, no. 2, pp. 111-119, 1988.

[18] J. Moors, "The meaning of kurtosis: Darlington reexamined," The American Statistician, vol. 40, no. 4, pp. 283-284, 1986.

[19] M. Khan, B. Sallberg, J. Nordberg and I. Claesson, "Noncoherent detection of impulse radio UWB signal based on fourth order statistics," in Proc. of IEEE International Conference on Ultra-wide Band, Vancouver, BC, Canada, Sept. 2009, pp. 824-828.

[20] A. Yang, H. Nie, Z. Xu and Z. Chen, "Modified kurtosis detection for impulse radio UWB signals," in Proc. 2013 IEEE Radio and Wireless Symposium (RWS2013), Austin, TX, USA, Jan. 2013, pp. 136-138.

[21] X. Feng, Z. Chen and Z. Xu, "Time-reversal source reconstruction with electromagnetic kurtosis," IEEE Trans. Antennas Propag., to be published. DOI: 10.1109/TAP.2021.3069523.

[22] J. de Rosny, G. Lerosey and M. Fink, "Theory of electromagnetic timereversal mirrors," IEEE Trans. Antennas Propag., vol. 58, no. 10, pp. 31393149, Oct. 2010. 
